\title{
Two-dimensional charged magneto-excitons: Magnetic translations and localization
}

\author{
A. B. Dzyubenkd* \\ Institut für Theoretische Physik, J.W. Goethe-Universität, 60054 Frankfurt, Germany
}

A. Yu. Sivachenko

The Weizmann Institute of Science, Rehovot 76100, Israel

(February 8, 2020)

\begin{abstract}
For composite complexes - quasi-two-dimensional (2D) charged magneto-excitons $X^{-}$- we propose a new exact classification of states, which is based on magnetic translations. We consider implications of this symmetry for magneto-optical transitions. It is shown, in particular, that in a translationally-invariant system with a simple valence band, the ground triplet $X_{t}^{-}$state is dark in interband transitions at finite magnetic fields $B$. This exact result calls for the re-interpretation of several previous theoretical studies. We consider the symmetry-breaking effects of spatial lateral confinement on internal transitions of charged complexes.
\end{abstract}

Recently, there has been considerable experimental and theoretical interest in charged excitons $X^{-}$and $X^{+}$ in magnetic fields $B$ in $2 \mathrm{D}$ systems. Experimentally, magneto-optical interband [1] and, more recently, intraband internal [2] transitions of charged excitons have been studied. Theoretically, the binding of charged excitons $X^{-}$has been considered in quantum dots [3,5], in a strictly-2D system in the high-magnetic field limit [ 4 , and in realistic quantum wells (QW's) at finite $B$ [6]. In all these theoretical works on charged excitons, the existing exact symmetry - magnetic translations - has not been identified. Some implications of this symmetry for internal transitions of charged complexes have been indicated recently in [2,7]. The aim of the present work is to establish and describe in some detail the underlying hidden symmetry and reveal its striking manifestations in interband and intraband magneto-optical transitions.

We consider a system of interacting particles of charges $e_{j}$ in a magnetic field $\mathbf{B}=(0,0, B)$ described by the Hamiltonian

$$
H=\sum_{j} \frac{\hat{\pi}_{j}^{2}}{2 m_{j}}+\frac{1}{2} \sum_{i \neq j} U_{i j}\left(\mathbf{r}_{i}-\mathbf{r}_{j}\right),
$$

here $\hat{\boldsymbol{\pi}}_{j}=-i \hbar \boldsymbol{\nabla}_{j}-\frac{e_{j}}{c} \mathbf{A}\left(\mathbf{r}_{j}\right)$ is the kinematic momentum operator of the $j$-th particle in $\mathbf{B}$ and $U_{i j}$ are the potentials of interactions that can be rather arbitrary. Dynamical symmetries of (1) are the following. In the symmetric gauge $\mathbf{A}=\frac{1}{2} \mathbf{B} \times \mathbf{r}$, there is the axial symmetry about the $z$-axis $\left[H, \hat{L}_{z}\right]=0$, where $\hat{L}_{z}=\sum_{j}\left(\mathbf{r}_{j} \times-i \hbar \boldsymbol{\nabla}_{j}\right)_{z}$. Therefore, the total angular momentum projection $M_{z}$, an eigenvalue of $\hat{L}_{z}$, is a good quantum number. In a uniform $\mathbf{B}$, the Hamiltonian (11) is also invariant under a group of magnetic translations whose generators are the components of the operator $\hat{\mathbf{K}}=\sum_{j} \hat{\mathbf{K}}_{j}$, where $\hat{\mathbf{K}}_{j}=\hat{\boldsymbol{\pi}}_{j}-\frac{e_{j}}{c} \mathbf{r}_{j} \times \mathbf{B}$ (see, e.g., [8]). $\hat{\mathbf{K}}$ is the exact integral of the motion: $[H, \hat{\mathbf{K}}]=0$. The components of $\hat{\mathbf{K}}$ and $\hat{\boldsymbol{\pi}}=\sum_{j} \hat{\boldsymbol{\pi}}_{j}$ commute in $\mathbf{B}$ as

$$
\left[\hat{K}_{x}, \hat{K}_{y}\right]=-\left[\hat{\pi}_{x}, \hat{\pi}_{y}\right]=-i \frac{\hbar B}{c} Q \quad, \quad Q \equiv \sum_{j} e_{j},
$$

while $\left[\hat{K}_{p}, \hat{\pi}_{q}\right]=0, p, q=x, y$. For neutral complexes (atoms, excitons, biexcitons) $Q=0$, and classification of states in $B$ are due to the continuous two-component vector - the 2D magnetic momentum $\mathbf{K}=\left(K_{x}, K_{y}\right)$. For charged systems the components of $\hat{\mathbf{K}}$ cannot be observed simultaneously. This determines the macroscopic Landau degeneracy of exact eigenstates of (1). For a dimensionless operator $\hat{\mathbf{k}}=\sqrt{c / 2 \hbar B|Q|} \hat{\mathbf{K}}$ we have $\left[\hat{k}_{x}, \hat{k}_{y}\right]=$ $-i Q /|Q|$. Therefore, $\hat{k}_{ \pm}=\left(\hat{k}_{x} \pm i \hat{k}_{y}\right) / \sqrt{2}$ are Bose raising and lowering ladder operators: $\left[\hat{k}_{+}, \hat{k}_{-}\right]=-Q /|Q|$. It follows then that $\hat{\mathbf{k}}^{2}=\hat{k}_{+} \hat{k}_{-}+\hat{k}_{-} \hat{k}_{+}$has discrete oscillator eigenvalues $2 k+1, k=0,1, \ldots$ Since $\left[\hat{\mathbf{k}}^{2}, H\right]=0$ and $\left[\hat{\mathbf{k}}^{2}, \hat{L}_{z}\right]=0$, the exact charged eigenstates of (11), in addition to the electron $S_{e}$ and hole $S_{h}$ spin quantum numbers, can be simultaneously labeled by the discrete quantum numbers $k$ and $M_{z}$. The labelling therefore is $\left|k M_{z} S_{e} S_{h} \nu\right\rangle$, where $\nu$ is a "principal" quantum number, which can be discrete (bound states) or continuous (unbound states forming a continuum) [7]. The $k=0$ states are Parent States (PS's) within a degenerate manifold. All other daughter states in each $\nu$-th family are generated out of the PS iteratively: for $Q<0$ $\left|k, M_{z}-k, S_{e} S_{h} \nu\right\rangle=\left(\hat{k}_{-}\right)^{k}\left|0, M_{z}, S_{e} S_{h} \nu\right\rangle / \sqrt{k !}$.

It is interesting to consider the following question: is it possible to construct a complete orthonormal basis with simultaneously fixed quantum numbers $M_{z}$ and $k$ (and $\left.S_{e}, S_{h}\right)$ ? The answer is positive. Let us demonstrate this on the example of the charged exciton $X^{-}$. The basis for the $X^{-}$with fixed values of $M_{z}$ and $S_{e}$ is of the form [9.2] $\phi_{n_{1} m_{1}}^{(e)}\left(\mathbf{r}_{e}\right) \phi_{n_{2} m_{2}}^{(e)}\left(\mathbf{R}_{e}\right) \phi_{N_{h} M_{h}}^{(h)}\left(\mathbf{r}_{h}\right)$ and includes differ- 
ent three-particle $2 e-h$ states such that the total angular momentum projection $M_{z}=n_{1}+n_{2}-m_{1}-m_{2}-N_{h}+M_{h}$ is fixed. Here $\phi_{n m}^{(e, h)}$ are the $e$ - and $h$ - single-particle factored wave functions in $B, n$ is the Landau-level (LL) quantum number, and $m$ the oscillator quantum number $\left[m_{z e(h)}={ }_{(-)}^{+}(n-m)\right]$. We use the electron relative and center-of-mass $(\mathrm{CM})$ coordinates: $\mathbf{r}_{e}=\left(\mathbf{r}_{e 1}-\mathbf{r}_{e 2}\right) / \sqrt{2}$ and $\mathbf{R}_{e}=\left(\mathbf{r}_{e 1}+\mathbf{r}_{e 2}\right) / \sqrt{2}$. Permutational symmetry requires that for electrons in the spin-singlet $S_{e}=1$ (triplet $S_{h}=1$ ) state the relative motion angular momentum $n_{1}-m_{1}$ should be even (odd). This basis complies with the axial symmetry about the $z$-axis and the permutational symmetry. To make it compatible with magnetic translations we shall perform a unitary transformation. To this end we introduce the intraLL ladder operators $B_{e}^{\dagger}$ and $B_{h}^{\dagger}$ (see, e.g., [9]), corresponding to the $\mathbf{R}_{e}$ and $\mathbf{r}_{h}$ degrees of freedom, respectively, and perform Bogoliubov canonical transformations $B_{e}^{\dagger} \rightarrow \tilde{B}_{e}^{\dagger}=S B_{e}^{\dagger} S^{\dagger}=u B_{e}^{\dagger}+v B_{h}, B_{h}^{\dagger} \rightarrow \tilde{B}_{h}^{\dagger}=$ $S B_{h}^{\dagger} S^{\dagger}=u B_{h}^{\dagger}+v B_{e}$, where $S=\exp \left\{\Theta\left(B_{e} B_{h}-B_{h}^{\dagger} B_{e}^{\dagger}\right)\right\}$ and $u=\operatorname{ch} \Theta, v=\operatorname{sh} \Theta$. Choosing th $\Theta=1 / \sqrt{2}$ we make $\hat{\mathbf{k}}^{2}=2 \tilde{B}_{e}^{\dagger} \tilde{B}_{e}+1$ diagonal in $\tilde{B}_{e}^{\dagger}$. Therefore, using transformed operators $\tilde{B}_{e}^{\dagger}$ and $\tilde{B}_{h}^{\dagger}$ operating on the new vacuum $|\tilde{0}\rangle=S|0\rangle=(\operatorname{ch} \Theta)^{-1} \exp \left\{-\operatorname{th} \Theta B_{h}^{\dagger} B_{e}^{\dagger}\right\}|0\rangle$ solves this problem. For a system of $N_{e}$ electrons and $N_{h}$ holes (with, e.g., $N_{e}>N_{h}$ ), the analogous transformation should involve the intra-LL $e$ - and $h$ - CM operators $B_{e}^{\dagger}$ and $B_{h}$ with th $\Theta=\sqrt{N_{h} / N_{e}}$.

Let us discuss now magneto-optical transitions of charged complexes. In the dipole approximation the photon momentum is negligibly small. Therefore, the quantum number $k$ should be conserved in intra- [2, ] and inter-band magneto-optical transitions. Let us establish this selection rule more formally. For internal intraband transitions the Hamiltonian of the interaction with the radiation of polarization $\sigma^{ \pm}$is of the form $\hat{V}^{ \pm} \sim \sum_{j} e_{j} \pi_{j}^{ \pm} / m_{j}$, where $\pi_{j}^{ \pm}=\pi_{j x} \pm i \pi_{j y}$. Conservation of $k$ follows from the commutativity $\left[\hat{V}^{ \pm}, \hat{\mathbf{K}}\right]=0$. Other selection rules in this case are conservation of spins and $\Delta M_{z}= \pm 1$ in the $\sigma^{ \pm}$polarization for the envelope function. For interband transitions the interaction with the radiation field is described by the luminescence operator $\hat{\mathcal{L}}=p_{\text {cv }} \int d \mathbf{r} \hat{\Psi}_{e}^{\dagger}(\mathbf{r}) \hat{\Psi}_{h}^{\dagger}(\mathbf{r})+$ H.c., where $p_{\text {cv }}$ is the momentum interband matrix element. Conservation of $k$ follows from $[\hat{\mathcal{L}}, \hat{\mathbf{K}}]=0$. Other selection rules for this case are conservation of spins and $\Delta M_{z}=0$ in the $\sigma^{ \pm}$ polarization for the envelope function (the Bloch parts change according to $\Delta m_{z}= \pm 1$ ). Conservation of $k$ constitutes a new exact selection rule. It can be formulated in a simple verbal way: Parents can talk only to parents, while daughters talk to daughters if and only if their parents do talk.

Let us consider implications of this selection rule for the interband transition - photoluminescence from the triplet ground state $X_{t}^{-} \rightarrow e_{n}^{-}+h \nu$ with the emission of the photon and the electron in the $n$-th LL in the final state. It turns out that simultaneous conservation of $M_{z}$ and $k$ in this transition makes it strictly prohibited: It is now well established [4, 6,7] that the triplet $X_{t}^{-}$ground PS with $k=0$ has $M_{z}=-1$ at finite $B>8 \mathrm{~T}$ and in quasi-2D QW's. On the other hand, an electron in the $n$-th LL, $e_{n}^{-}$, has $M_{z}=n-k$ (the oscillator quantum number $m=k$ ). We see that $\Delta k=0, \Delta M_{z}=0$ cannot be satisfied for transition to any electron LL $n$ if the $X^{-}$PS has $M_{z}<0$. This means that the ground triplet state $X_{t}^{-}$is optically dark in interband transitions (note that the singlet ground state $X_{s}^{-}$has $M_{z}=0$ and is optically active). In the $2 \mathrm{D}$ high- $B$ limit this also follows [4] from the so called "hidden symmetry" in $e-h$ systems 10]. We stress that our result is much more general. Indeed, quasi-2D effects, $e-h$ asymmetry, admixture of higher LL's (finite $B$-effects) break neither axial nor translational symmetry. Therefore, even in the presence of these effects, the triplet stays dark - as long as the ground $X_{t}^{-}$PS has $M_{z}<0$. This exact result calls for re-interpretation of several previous theoretical works on optical properties of the triplet $X_{t}^{-}$ground state [4] 6. Very small but finite oscillator strengths of the $X_{t}^{-}$ ground state obtained [4,6] should in fact be considered as artifacts coming from the finite-size effects and/or inaccuracy in numerics. Large oscillator strengths of the unconfined $X_{t}^{-}$ground state with $M_{z}<0$ [5] is not compatible with the exact selection rule and is an error. On the other hand, finite and large oscillator strengths of the spatially confined $X_{t}^{-}$ground state [3] (see also [5]) results from the breaking of translational invariance. The question now remains why in fact the $X_{t}^{-}$ground state is visible in experiment. One of the possibilities is localization of charged excitons in real samples. This would imply that the oscillator strength of the triplet ground state should vary from sample to sample. Another possibility comes from intrinsic effects and is associated with the complex character of the valence band. Finally, the rearrangement of the ground $X_{t}^{-}$state with decreasing $B$ can in principle occur: with decreasing separation between LL's the ground $X_{t}^{-}$state could jump from $M_{z}=-1$ to $M_{z}=0$. This would imply that (1) there exist some critical field $B_{c}$ above which the $X_{t}^{-}$state abruptly becomes dark, and (2) the experimental situation corresponds to $B<B_{c}$. Numerical calculations suggest that the latter scenario appears not to be very plausible. More theoretical work is needed to clarify the situation here.

We turn now to intraband transitions of charged magneto-excitons. Such transitions in translationally invariant systems are discussed theoretically in [2], where also experimental results for bound-to-continuum transitions are reported and comparison between theory and experiment is made. It was shown [2,7] that the boundto-bound transition from the triplet ground state to a more stronger bound state associated with the next 


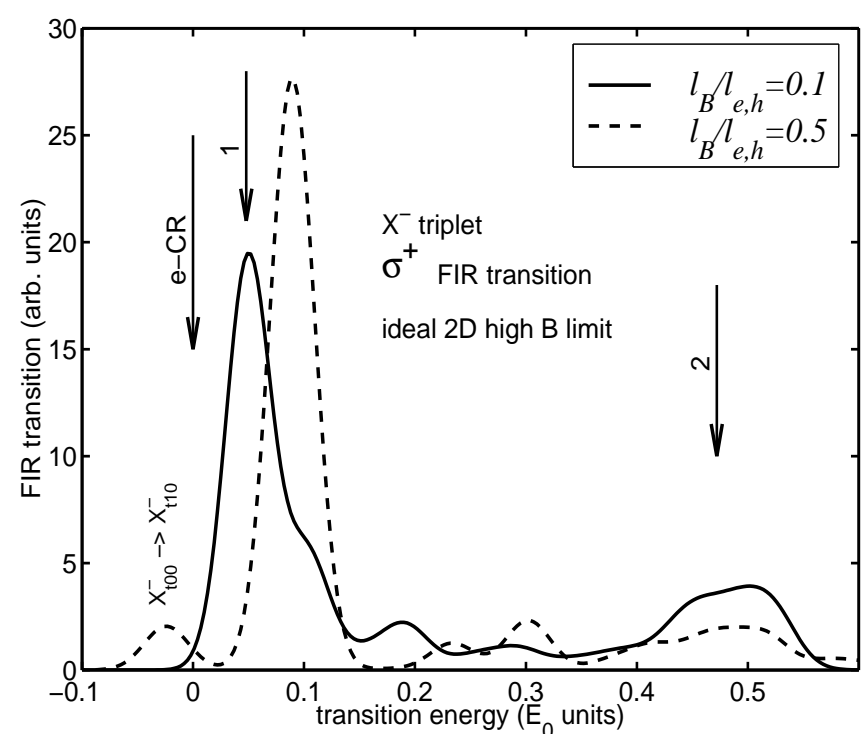

Fig. 1. Internal intraband transitions from the triplet $X_{t 00}^{-}$ground state in the presence of parabolic lateral confinement parametrized by lengths $l_{e}=l_{h}=l_{\mathrm{e}, \mathrm{h}}$. Strictly $2 \mathrm{D}$ system in the high- $B$ limit is considered. Two cases of lateral confinement $l_{B} / l_{\mathrm{e}, \mathrm{h}}=0.1$ and $l_{B} / l_{\mathrm{e}, \mathrm{h}}=0.5$ are shown; $l_{B}=(\hbar c / e B)^{1 / 2}$. Energies are given in units of $E_{0}=\sqrt{\pi / 2} e^{2} / \epsilon l_{B}$ relative to the confinement-renormalized $e$-CR energy shown by vertical arrow. Vertical arrows 1 and 2 correspond, for the weak-confinement regime $l_{B} / l_{\mathrm{e}, \mathrm{h}}=0.1$, to the bound-to-continuum transitions discussed in unconfined systems in 还,

electron LL, $X_{t 00} \rightarrow X_{t 10}$, is prohibited. Indeed the $X_{t 00}$ PS has $M_{z}=-1$, while the $X_{t 10}$ PS has $M_{z}=1$, so that the usual selection rule $\Delta M_{z}= \pm 1$ cannot be satisfied. It is interesting to study how localization of charged excitons breaks translational invariance and relaxes the $k$-conservation rule. We model localization by the in-plane lateral spatial confinement, which is assumed parabolic for electrons and holes $V_{e}=\frac{1}{2} m_{e} \omega_{e}^{2}\left(x_{e}^{2}+y_{e}^{2}\right)$, $V_{h}=\frac{1}{2} m_{h} \omega_{h}^{2}\left(x_{h}^{2}+y_{h}^{2}\right)$ with characteristic lengths $l_{e}=$ $\left(\hbar / 2 m_{e} \omega_{e}\right)^{1 / 2}$ and $l_{h}=\left(\hbar / 2 m_{h} \omega_{h}\right)^{1 / 2}$, chosen such that $l_{e}=l_{h} \equiv l_{\mathrm{e}, \mathrm{h}}$ This model of confinement has been previously considered for neutral [11] and charged [3,5] magnetoexcitons. Here we present results for a strictly-2D situation in the limit of high magnetic fields (no Landau level mixing). The spectra of internal transitions from the triplet ground state $X_{t 00}$ to states in the next electron LL for two regimes of lateral confinement are shown in Fig. 1. Energies in Fig. 1 are given relative to the confinement-renormalized electron cyclotronresonance $(e-\mathrm{CR}) \hbar \tilde{\omega}_{\mathrm{ce}}=\hbar \omega_{\mathrm{ce}}\left(\sqrt{1+\left(l_{B} / l_{\mathrm{e}, \mathrm{h}}\right)^{4}}+1\right) / 2$, $l_{B}=(\hbar c / e B)^{1 / 2}$. In the regime of weak confinement $l_{B} / l_{\mathrm{e}, \mathrm{h}}=0.1$ the spectra practically reproduce the unconfined 2D case [7]. For the intermediate confinement $l_{B} / l_{\mathrm{e}, \mathrm{h}}=0.5$ the bound-to-bound $X_{t 00}^{-} \rightarrow X_{t 10}^{-}$transition, which is prohibited in translationally-invariant systems, develops below the $e$-CR. Development of such peak is a tell-tale mark of localization of charged triplet excitons.

In conclusion, we have studied the exact symmetry magnetic translations - for charged excitons in $B$ and established its consequences for interband and intraband optical transitions. In particular, we have shown that in translationally invariant quasi-2D system with a simple valence band the triplet ground state $X_{t}^{-}$is dark in intraband transitions at finite fields $B$. We have shown that in the presence of symmetry-breaking effects the intraband bound-to-bound triplet transition develops below the electron cyclotron resonance. This suggests a method of studying localization of charged excitons.

This work was financially supported in part by the DFG grant Os $98 / 5$. ABD is grateful to the Alexander von Humboldt Stiftung for research support.

* on leave from General Physics Institute, RAS, Moscow 117942, Russia.

[1] See, e.g., K. Kheng, R.T. Cox, Merle Y. d'Aubigné et al. Phys. Rev. Lett. 71, 1752 (1993); G. Finkelstein, H. Shtrikman, and I. Bar-Joseph, Phys. Rev. Lett. 74, 976 (1995); A.J. Shields, M. Pepper, M.Y. Simmons, and D.A. Ritchie, Phys. Rev. B 52, 7841 (1995); D. Gekhtman, E. Cohen, Arza Ron, and L.N. Pfeiffer, Phys. Rev. B 54, 10320 (1996), D.R. Yakovlev, V.P. Kochereshko, R. Suris et al. Phys. Rev. Lett. 79, 3974 (1997) and references therein.

[2] A.B. Dzyubenko, A.Yu. Sivachenko, H.A. Nickel, T.M. Yeo, G. Kioseoglou, B.D. McCombe, and A. Petrou, cond-mat/9907146; Proceedings of EP2DS-13, to be published in Physica E.

[3] A. Wojs and P. Hawrylak, Phys. Rev. B 51, 10880 (1995).

[4] J.J. Palacios, D. Yoshioka, and A.H. MacDonald, Phys. Rev. B 54, R2296 (1996).

[5] J.R. Chapman, N.F. Johnson, and V.N. Nicopoulos, Phys. Rev. B 55, R10 221 (1997); 57, 1762.

[6] D.M. Whittaker and A.J. Shields, Phys. Rev. B 56, 15185 (1997).

[7] A.B. Dzyubenko and A.Yu. Sivachenko, cond-mat/9902086.

[8] J.E. Avron, I.W. Herbst, and B. Simon, Annals of Phys. 114, 431 (1978).

[9] A.B. Dzyubenko, Sov.Phys.-Solid State 311885 (1989); Solid State Commun. 74409 (1990); Phys. Lett. A 173, 311 (1993).

[10] I.V. Lerner and Yu.E. Lozovik, JETP 53, 763 (1981)]; A.B. Dzyubenko and Yu.E. Lozovik, Sov. Phys. Solid State 25, 874 (1983); 26, 938 (1984); A.H. MacDonald and E.H. Rezayi, Phys. Rev. B 42, 3224 (1990).

[11] A.B. Dzyubenko and A.Yu. Sivachenko, J. Phys. IV (Paris), 3, 381 (1993). 